\title{
ECLETICA
}

www.scielo.br/eq

Volume 30, número 2, 2005

\section{Sistema TS-FF-AAS com chama acetileno-ar como alternativa em relação à chama acetileno-óxido nitroso em FAAS na determinação de estanho}

\author{
F. A. Lobo ${ }^{1}$, A. C.Villafranca ${ }^{1}$, A. P. de Oliveira ${ }^{1}$, M. de Moraes ${ }^{1 *}$ \\ ${ }^{1}$ Instituto de Química - Universidade Estadual Paulista, UNESP, \\ CP 355, CEP 14801-970 Araraquara -SP \\ mermorae@.iq.unesp.br
}

\begin{abstract}
Resumo: Este trabalho propõe o desenvolvimento de um procedimento analítico para a determinação em linha de estanho em soluções analíticas empregando a técnica de espectrometria de absorção atômica com nebulização térmica em tubo metálico aquecido na chama (TS-FF-AAS) como alternativa ao uso da chama de óxido nitroso-acetileno em espectrometria de absorção atômica, cujo comburente apresenta um custo elevado frente ao ar comprimido. Foram avaliados parâmetros para a otimização do sistema, tais como: vazão do carregador (ar), volume de amostra injetada e concentração do ácido (HCl). Foi construída curva analítica linear $[\mathrm{A}=-0,00163+0,00319[\mathrm{Sn}],(\mathrm{r}=0,9998)]$ no intervalo de 10 a $80 \mathrm{mg} \mathrm{Sn} \mathrm{L}^{-1}$. O limite de detecção, o desvio padrão relativo $(n=12)$ e a freqüência analítica foram: $1,7 \mathrm{mg} \mathrm{L}^{-1} \mathrm{Sn}, £ 2,7 \%$ and 120 $\mathrm{h}^{-1}$ respectivamente. O sistema TS-FF-AAS é uma alternativa para a determinação de $\mathrm{Sn}$.
\end{abstract}

Palavras-chave: TS-FF-AAS; estanho; tubo metálico.

\section{Introdução}

O monitoramento de estanho é de extrema importância do ponto de vista da saúde pública, pois é considerado o contaminante prioritário devido ao aumento do uso do mesmo na embalagem de alimentos enlatados [1].

A espectrometria de absorção atômica (AAS) pode ser empregada na determinação quantitativa de muitos elementos (metais e semi-metais) em uma ampla variedade de amostras alimentícias, biológicas, ambientais, geológicas, entre outras. O princípio da técnica baseia-se na medida da absorção da radiação eletromagnética, proveniente de uma fonte de radiação, por átomos gasosos no estado fundamental. O processo de formação de átomos gasosos no estado fundamental, denominado atomização, pode ser obtido via chama, via eletrotérmica, ou por reação química específica, como a geração de vapor frio de $\mathrm{Hg}$. Atomizadores eletrotérmicos compreendem principalmente os tubos de grafite, filamentos de tungsténio, tubos de quartzo (para a atomização de hidretos) e tubos metálicos ou cerâmicos. A espectrometria de absorção em chama (FAAS) é a mais utilizada para análises elementares em níveis de $\mathrm{mg} \mathrm{L}^{-1}$ [2].

O estanho pode ser determinado por FAAS, e recomenda-se que se utlilize a chama acetilenoóxido nitroso, que é uma chama mais quente podendo atingir cerca de $3000^{\circ} \mathrm{C}$, a qual minimiza as possíveis interferências nos processos de atomização do analito. No entanto, esta chama sendo mais quente expõe o equipamento e as conexões a altas temperaturas ocasionando um maior desgaste dos mes- 
mos, além do comburente apresentar um custo elevado frente ao ar comprimido. Outros sistemas foram desenvolvidos para a determinação de estanho, tais como a geração de hidretos em espectrometria de absorção atômica, porém para o estanho observa-se picos duplos e falsos, efeitos de memória, deposição de estanho no tubo de quartzo, revolatilização e adsorção do estanho na superfície da cela de quartzo, elevado tempo de purga do hidreto gerado e outros efeitos adversos [3]. Uma alternativa interessante para a determinação de estanho é a espectrometria de absorção atômica com nebulização térmica em tubo aquecido na chama (TS-FF-AAS) [4,5].

O Thermospray (TS) foi originalmente desenvolvido por Vestal et al. em 1978 [5] como uma interface entre a cromatografia líquida e a espectrometria de massa. Em espectrometria atômica, o aquecimento do tubo era feito eletricamente para manter a temperatura constante, e desta forma limitava o uso da técnica a poucos elementos. Em contraste Gaspar e Berndt [5] propõem o TS-FF-AAS, no qual um tubo metálico é posicionado sobre a chama do espectrômetro de absorção atômica funcionando como um reator. $\mathrm{O}$ líquido é transportado através de um capilar metálico conectado ao tubo aquecido pela chama. $\mathrm{O}$ capilar é aquecido simultaneamente com o tubo pela chama do espectrômetro de absorção atômica. Alcançando a extremidade quente do capilar o líquido vaporiza-se parcialmente e um aerossol é formado. Por fim, o aerossol é vaporizado dentro do tubo, formando a nuvem atômica que absorve a radiação proveniente da lâmpada. Assim o TSFF-AAS foi considerado efetivamente como uma interface entre cromatografia líquida de alta resolução (HPLC) acoplada a FAAS através de um sistema de injeção em fluxo [5]. A partir deste trabalho surgiram várias determinações utilizando a espectrometria de absorção atômica com forno aquecido na chama (TS-FF-AAS) $[6,7,8,9,10$, $11,12]$.

O objetivo deste trabalho é desenvolver uma alternativa de procedimento analítico para a determinação em linha de estanho empregando o sistema de espectrometria de absorção atômica com forno aquecido na chama acetileno-ar (TS-FF-AAS) em relação à chama acetileno-óxido nitroso convencionalmente utilizada em FAAS.

\section{Procedimento Experimental}

\section{Instrumentos e acessórios}

Os instrumentos utilizados foram o espectrômetro de absorção atômica com atomizador de chama, Perkin-Elmer, modelo AAnalyst 100, lâmpada de catodo oco de $\operatorname{Sn}(1=224,6 \mathrm{~nm}$, fenda $=0,2 \mathrm{~nm}$ e $\mathrm{i}=20 \mathrm{~mA}$ ), vazão dos gases na proporção 4:2 (ar:acetileno), lâmpada de deutério para correção de background (fundo); balança analítica Sartorius BL 2105 e bomba peristáltica Ismatec, Modelo ICP 8.

No sistema TS-FF-AAS foi utlizada uma válvula de injeção Rheodyne RE9725, tubos de PEEK, capilar cerâmico OMEGA TRX-164116 (OMEGATITE ${ }^{\circledR} 450$ ) com as seguintes características: capilar isolante de termopar de $\mathrm{Al}_{2} \mathrm{O}_{3}>$ $99,8 \%$, que suporta temperaturas até $1900{ }^{\circ} \mathrm{C}$, com $\varnothing_{\text {ext }}=1,6 \mathrm{~mm}$ e dois orifícios com $\varnothing_{\mathrm{int}}=0,4 \mathrm{~mm}$ (este capilar produz melhores resultados que um capilar de aço inox (HPLC), pois produz menor ruído nos sinais de absorbância), tubo metálico de super-liga Ni-Cr $\left(\right.$ Inconel $\left.^{\circledR}\right)$, comprimento $=100$ $\mathrm{mm} \operatorname{com} \varnothing_{\text {int }}=10,0 \mathrm{~mm}$ e $\varnothing_{\text {ext }}=12,0 \mathrm{~mm}, 6$ orifícios com $\varnothing=2,5 \mathrm{~mm}$, perpendiculares a um orifício $\operatorname{com} \varnothing=2,0 \mathrm{~mm}$.

A aquisição de dados foi feita através do software MQDOS, Microquímica e os valores de absorbância foram proporcionais à altura dos sinais transientes.

As medidas de temperatura no interior do tubo foram realizadas de duas formas. A primeira medida foi feita com o termopar com junta aterrada, posicionado junto ao tubo metálico, na direção do orifício onde se coloca o capilar cerâmico de introdução da amostra no tubo atomizador. A temperatura medida foi $983 \pm 1^{\circ} \mathrm{C}$ para o tubo metálico [13].

Com o termopar de junta exposta, posicionado juntamente com o capilar cerâmico dentro do tubo, mediu-se a temperatura no interior do mesmo. $\mathrm{O}$ tubo apresentou intervalo de temperatura entre $1030^{\circ} \mathrm{C} \mathrm{a} 1060^{\circ} \mathrm{C} \mathrm{em}$ seu interior e, ficou totalmente rubro sobre a chama [13].

Quando injeta-se 50 ì de $\mathrm{HNO}_{3}(\sim 0,1 \mathrm{~mol}$ $\left.\mathrm{L}^{-1}\right)$ a uma vazão de aproximadamente $1,5 \mathrm{~mL} \mathrm{~min}^{-}$ ${ }^{1}$ ocorre um abaixamento da temperatura de cerca de $50^{\circ} \mathrm{C}$, devido ao resfriamento do tubo pela solução. Entretanto, rapidamente volta aos seus intervalos máximos de temperatura [13]. 


\section{Reagentes e soluções}

A solução estoque de estanho $1000 \mathrm{mg} \mathrm{L}^{-1}$ foi preparada por pesagem do Sn metálico Carlo Erba 99\%, dissolvida em HCl (MERCK) e logo em seguida foi padronizada [14]. Soluções padrões de estanho foram preparadas diariamente por diluições sucessivas da solução estoque.

\section{Montagem do sistema TS-FF-AAS}

O sistema TS-FF-AAS montado no laboratório está representado na Figura 1 [13].
$\mathrm{Na}$ literatura afirma-se que o tubo de Inconel ${ }^{\circledR}$ deve ser colocado sobre a cabeça do queimador somente depois que a chama estiver acesa por existir a possibilidade de explosão no interior do tubo devido ao acúmulo de gás no seu interior. Neste trabalho, o sistema TS-FF-AAS foi montado primeiro e, posteriormente a chama do espectrômetro, foi acesa imediatamente após a abertura dos gases justamente para evitar um possível acúmulo de gás no interior do tubo. Este procedimento facilita a colocação do tubo sobre a cabeça do queimador, que é feito com a chama apagada [13].

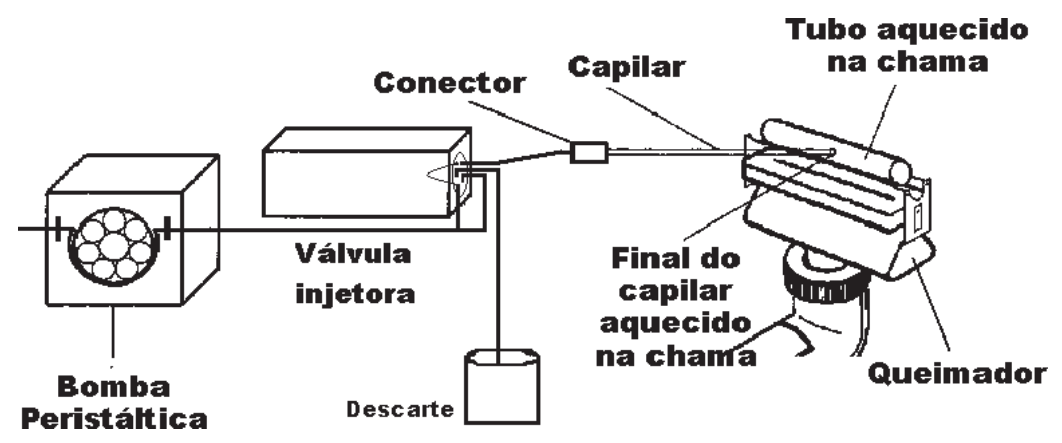

Figura 1. Arranjo esquemático do sistema TS-FF-AAS [13].

Em todo o sistema TS-FF-AAS foi utilizado um volume discreto de amostra, injetado no fluxo do carregador ar, pois segundo estudos da literatura utilizando-se soluções há uma maior diluição e dispersão da amostra [7,13].

A introdução da amostra no sistema TS-FFAAS (Figura 1) é feita por uma válvula manual Rheodyne, e em seguida a amostra é transportada por um carregador (ar) até o tubo capilar cerâmico. Como o capilar cerâmico é aquecido simultaneamente com o tubo metálico (reator), o líquido é parcialmente vaporizado, formando thermospray e ao chegar no tubo metálico, ocorre a atomização, gerando sinais transientes, que foram captados e armazenados por um software adequado. A altura dos sinais transientes foi utilizada como parâmetro analítico de medida.

Otimização da vazão do carregador, do volume de amostra e da concentração de ácido clorídrico

Foi avaliada a influência da variação da vazão do carregador $\left(0,6\right.$ a 6,0 $\left.\mathrm{mL} \mathrm{min}^{-1}\right)$, do volume de amostra $(20,50,100$ e $200 \mathrm{~mL})$ e da concentração

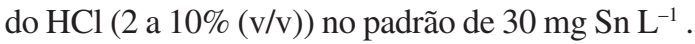

\section{Construção de curva analítica}

Após a otimização do sistema foi construída uma curva analítica no intervalo de 10 a $80 \mathrm{mg} \mathrm{Sn}$ $\mathrm{L}^{-1}$, e o limite de detecção (LD) foi determinado com base nas 12 leituras do branco.

\section{Resultados e Discussão}

Otimização da vazão do carregador, do volume de amostra e da concentração de ácido clorídrico

Ao injetar o padrão de $30 \mathrm{mg} \mathrm{Sn} \mathrm{L}^{-1}$ no fluxo carregador de ar, observou-se picos repetitivos. Ao injetar solução de $\mathrm{HCl} 2 \%$ (v/v) como carregador, a linha base subiu (aumento esperado, devido a injeção do branco) e com o passar do tempo a linha base caiu devido o resfriamento do tubo metálico. O resfriamento do tubo foi significativo, pois ao injetar o padrão não foi possível obter nenhum si- 
nal transiente, concluindo que a temperatura atingida pelo tubo não foi suficiente para atomizar o analito que ficou disperso na solução carregadora. Desta forma confirmou-se estudos anteriores de que ao usar ar (ou gás) como carregador evita-se a diluição e dispersão da amostra. Todas as análises foram feitas usando ar como carregador, não apenas porque reduz o custo de se usar uma solução como carregador e a quantidade de rejeito, mas também torna possível usar o sistema TS-FF-AAS para determinar estanho, que não seria possível se o carregador fosse solução.

A influência da vazão do carregador (ar) nos valores de absorbância de $50 \mathrm{~mL}$ do padrão de 30 $\mathrm{mg} \mathrm{Sn} \mathrm{L} \mathrm{em} \mathrm{HCl}^{-1} \%$ (v/v) foi estudado de $0,6 \mathrm{a}$ $6,0 \mathrm{~mL} \mathrm{~min}^{-1}$, como ilustra Figura 2.

Observando-se a Figura 2, verifica-se que para vazões baixas do carregador os valores de absorbância são baixos por que a amostra chega lentamente no atomizador, o tempo de medida é aumentado consideravelmente ocorrendo vaporização imprevisível e irregular. Portanto à medida que a vazão cresce, os valores de absorbância também crescem, pois vai gerando uma vaporização mais homogênea da amostra [5,9]. Este aumento ocorre até a vazão do carregador $3,0 \mathrm{~mL} \mathrm{~min}^{-1}$ onde se tem o máximo do valor de absorbância. Para vazões do carregador maiores que $3,0 \mathrm{~mL} \mathrm{~min}^{-1} \mathrm{o}$ tempo de residência do líquido na parte aquecida do capilar cerâmico é diminuído consideravelmente,

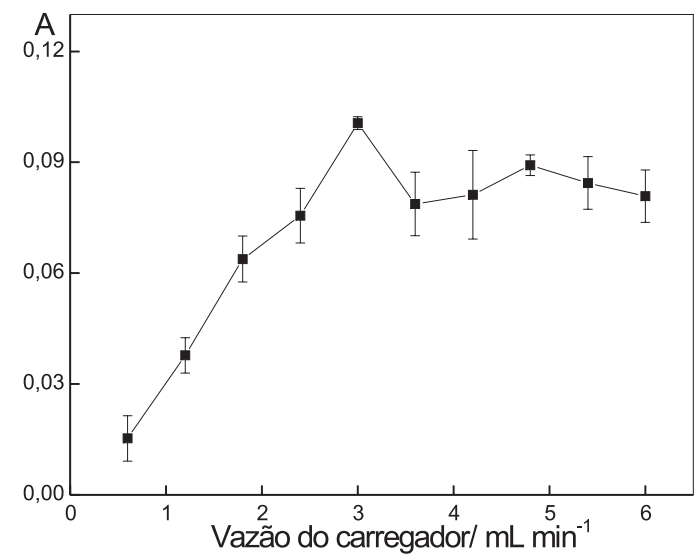

Figura 2. Influência do carregador (ar) sobre as absorbância de $50 \mathrm{~mL}$ da solução de $30 \mathrm{mg} \mathrm{L}^{-1}$ de $\mathrm{Sn}$ em $\mathrm{HCl} 2 \%$ (v/v). diminuindo o tempo para a evaporação do líquido e assim a amostra não chega como vapor/aerossol e sim como um fluxo contínuo do líquido. A temperatura no interior do tubo diminui rapidamente mudando a sua coloração de rubro brilhante para cinza opaco e pode ser observado que algumas gotículas saem para fora do tubo atomizador. Assim não se observa aumento significativo dos valores de absorbância, e estes variam muito, levando a altos valores de estimativa do desvio padrão. A vazão de 3,0 $\mathrm{mL} \mathrm{min}^{-1}$ foi escolhida como a melhor vazão devido ao maior valor de absorbância e ao menor valor da estimativa do desvio padrão.

$\mathrm{O}$ volume de amostra foi estudado variandose de 50 a $200 \mathrm{~mL}$ na vazão de $3,0 \mathrm{~mL} \mathrm{~min}^{-1}$ do carregador e os resultados são mostrados na Figura 3.

Observando-se a Figura 3, verifica-se que o volume de amostra de $20 \mathrm{~mL}$ apresenta valor de absorbância significativo, entretanto baixo, devido ao volume de amostra ser também baixo. Ao usar 50 $\mathrm{mL}$ de amostra houve um aumento significativo na intensidade do sinal de absorbância, pois ao aumentar o volume de amostra que é introduzida no atomizador do espectrômetro de absorção atômica, a população de átomos no volume de absorção também aumenta [8], sendo que para estes dois volumes, a estimativa do desvio padrão foi relativamente baixa, mostrando que há uma boa repetibildade nas medidas experimentais. Ao usar $100 \mathrm{~mL}$ de amostra, houve um pequeno resfriamento do capilar

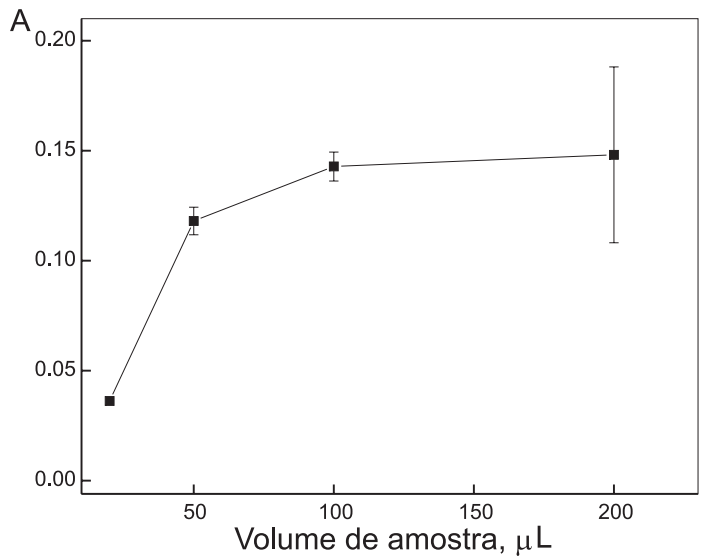

Figura 3. Influência do volume de amostra sobre as absorbâncias da solução de $30 \mathrm{mg} \mathrm{L}^{-1}$ de $\mathrm{Sn}$ em $\mathrm{HCl}$

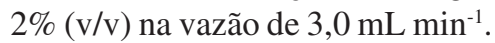


cerâmico, e conseqüentemente do tubo, não ocorrendo um aumento significativo nos valores de absorbância. Com 200 mL de amostra, o capilar cerâmico e o tubo resfriam muito e não há uma formação homogênea do thermospray, pois ocorre formação irregular de gotículas que dispersam a radiação, (possivelmente em grande extensão, que o corretor com lâmpada de deutério não é suficiente para corrigir totalmente o fundo gerado), o que leva a uma atomização imprevisível, causando um valor da estimativa do desvio padrão muito alto. Usando ar como carregador, o volume de $50 \mathrm{~mL}$ de amostra foi escolhido para as determinações seguintes, devido a maior homogeneidade na atomização, apresentando valor significativo de absorbância para o padrão de $30 \mathrm{mg} \mathrm{L}^{-1}$, com pequeno valor da estimativa do desvio padrão.

A Figura 4 mostra a influência da concentração do ácido $\mathrm{HCl}$ no intervalo de 2 a $10 \%$ (v/v) sobre os sinais de absorbância de $50 \mathrm{~mL}$ do padrão de $30 \mathrm{mg} \mathrm{L}^{-1}$ de $\mathrm{Sn}$ na vazão de $3,0 \mathrm{~mL} \mathrm{~min}^{-1}$ do carregador (ar).

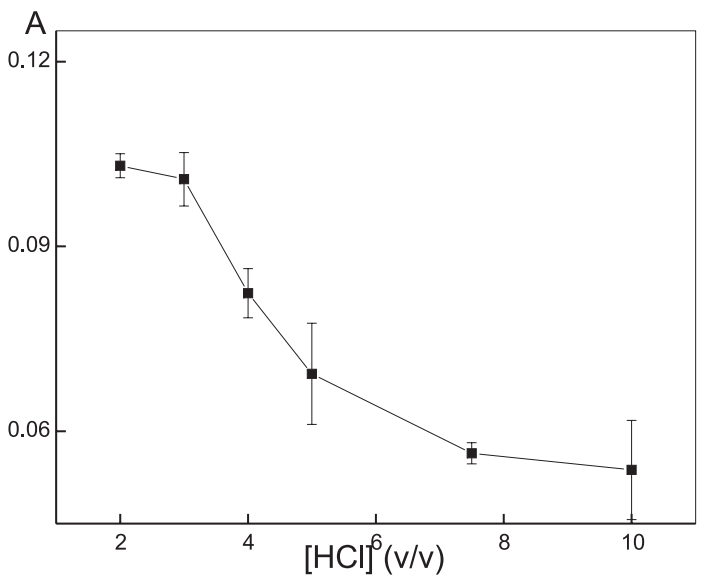

Figura 4. Influência da acidez sobre as absorbância da solução de $30 \mathrm{mg} \mathrm{L}^{-1}$ de $\mathrm{Sn}$ em diferentes concentrações de $\mathrm{HCl}$ em \% (v/v).

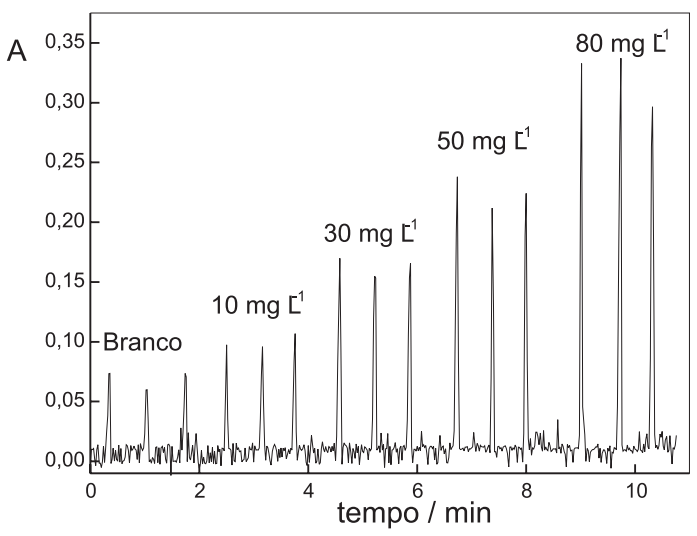

(a)

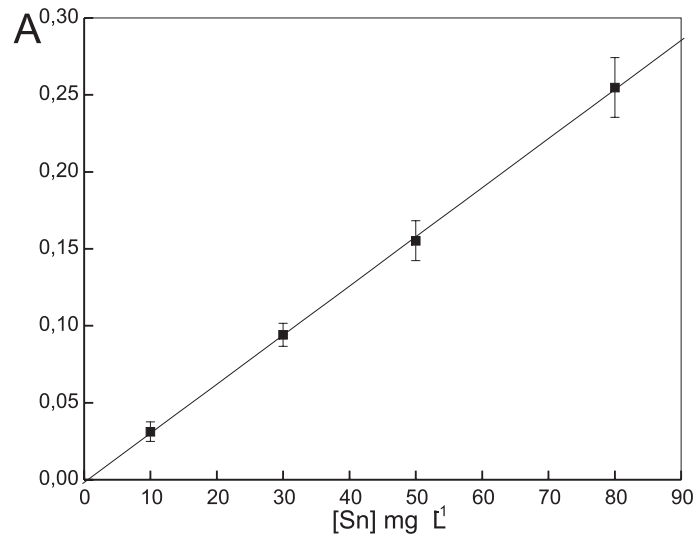

(b)

Figura 5. (a) Sinais transientes de estanho obtidos por TS-FF-AAS, no intervalo de 10 a $80 \mathrm{mg} \mathrm{L}^{-1} \mathrm{em} \mathrm{HCl}$ 2\% (v/v). (b) Equação ajustada: A=- 0,00163 + 0,00319[Sn], $r=0,9998$. 
Os valores de absorbância do padrão de 30 $\mathrm{mg} \mathrm{L}^{-1} \mathrm{Sn}$ diminuem consideravelmente para concentrações de $\mathrm{HCl}$ maiores que $3 \%$ (v/v) pois, provavelmente há perdas na atomização devido ao analito estar suceptível a uma maior volatilidade. Assim a acidez selecionada foi a de $2 \%$ (v/v), devido ao maior valor de absorbância e ao menor valor da estimativa do desvio padrão.

\section{Construção da curva analítica}

A Figura 5 ilustra os resultados obtidos para a curva analítica no intervalo de concentração de 10 a $80 \mathrm{mg} \mathrm{L}^{-1}$ de estanho em $\mathrm{HCl} 2 \%$ (v/v) usando as condições do sistema TS-FF-AAS otimizado.

A Figura 5 mostra que os sinais transientes foram repetitivos, e a curva analítica obtida apresentou-se linear no intervalo de concentração estudado.

A Figura 6 mostra os sinais transientes $(n=12)$ para a solução do branco $\mathrm{HCl} 2 \%$ (v/v).

Na Tabela 1 estão apresentadas as características analíticas otimizadas para a determinação de Sn por TS-FF-AAS, usando o tubo metálico como cela de atomização.

A Figura 6 mostra que houve boa repetibilidade na intensidade dos sinais transientes para o branco com o sistema otimizado, obtendo baixo limite de detecção como mostra o resultado da Tabela 1. As características analíticas otimizadas apresentadas na Tabela 1, mostram que foi possível obter uma curva analítica linear no intervalo de 10 a $80 \mathrm{mg} \mathrm{L}^{-1} \mathrm{de}$ Sn em $\mathrm{HCl} 2 \%(\mathrm{v} / \mathrm{v})$, com boa linearidade $(\mathrm{r}=0,9998)$.

O sistema pode ser considerado sensível, pois apresentou concentração característica igual a 1,4 $\mathrm{mg} \mathrm{L}^{-1}$, empregando a chama alimentada por acetileno-ar é rápido, possibilitando 120 determinações hora.

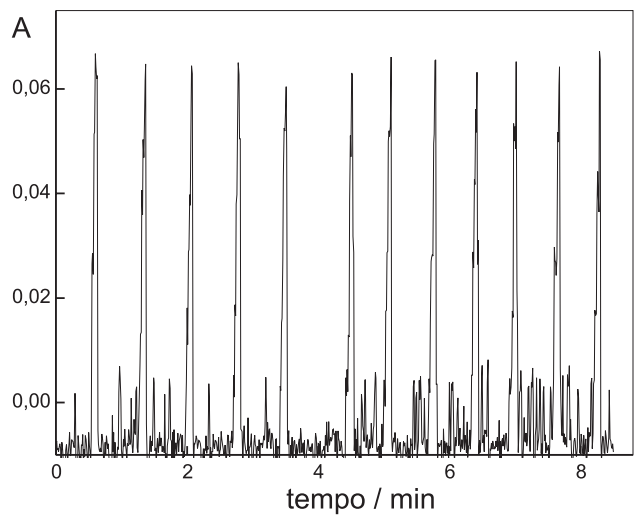

Figura 6. Sinais transientes obtidos por TS-FF-AAS, para 12 leituras do $\mathrm{HCl} 2 \%$ (v/v).

Tabela 1. Características analíticas otimizadas para a determinação de estanho por TS-FF-AAS, no intervalo de 10 a $80 \mathrm{mg} \mathrm{L}^{-1}$ em HCl 2\% (v/v), usando-se o tubo metálico como cela de atomização e FAAS com chama $\mathrm{C}_{2} \mathrm{H}_{2}-\mathrm{N}_{2} \mathrm{O}$.

\begin{tabular}{ccc}
\hline Características analíticas & TS-FF-AAS & FAAS - \\
\hline Desvio padrão relativo & $\mathrm{C}_{2} \mathrm{H}_{2}-\mathrm{N}_{2} \mathrm{O}[15]$ \\
$(\mathrm{n}=12, \mathrm{HCl} 2 \%(\mathrm{v} / \mathrm{v}))$ & $\leq 2,7$ & $\leq 13,3$ \\
Concentração Característica & & \\
$\left(\mathrm{mg} \mathrm{L}^{-1}\right)$ & 1,4 & 3,1 \\
Freqüência analítica $\left(\mathrm{h}^{-1}\right)$ & 120 & 360 \\
Equação da curva analítica & $\mathrm{A}=-0,0016+0,0032[\mathrm{Sn}]$ & $\mathrm{A}=0,016+0,0014[\mathrm{Sn}]$ \\
Coeficiente de correlação $(\mathrm{r})$ & 0,9998 & 0,9995 \\
Volume de amostra $(\mu \mathrm{L})$ & 50 & $\sim 1000$ \\
Vazão $\left(\mathrm{mL} \mathrm{min}^{-1}\right)$ & 3,0 (carregador) & 7,6 (nebulizador) \\
LD $\left(\mathrm{mg} \mathrm{L} \mathrm{L}^{-1}\right)$ & 1,7 & 9,6 \\
\hline
\end{tabular}


A Tabela 1 mostra que o sistema TS-FF-AAS empregando chama acetileno-ar apresenta melhor desempenho analítico que a espectrometria de absorção atômica com atomizador de chama (FAAS) alimentada por acetieno-óxido nitroso. $\mathrm{O}$ desvio padrão relativo é cerca de cinco vezes menor, a sensibilidade da curva analítica é cerca de duas vezes maior e o limite de detecção é mais de cinco vezes menor que os respectivos parâmetros obtidos por FAAS com chama acetileno-óxido nitroso.

\section{Conclusões}

Ao otimizar o sistema TS-FF-AAS, usando o tubo metálico como cela de atomização para a determinação de Sn foi possível observar os parâmetros que influenciam no processo de atomização deste analito e as condições que são ideais para o sistema na avaliação dos padrões no intervalo de 10 a $80 \mathrm{mg} \mathrm{L}^{-1}$, preparados em $\mathrm{HCl} 2 \%$ (v/v). O sistema TS-FF-AAS é simples, promissor e de baixo custo analítico, pois permite a redução do consumo de amostras (apenas $50 \mathrm{~mL}$ ) e reagentes e da geração resíduos. O procedimento analítico desenvolvido para a determinação de estanho em soluções é de fácil operação, tem alta frequiência analítica $\left(120 \mathrm{~h}^{-1}\right)$ e é uma alternativa, com melhor desempenho analítico, ao emprego do comburente óxido nitroso em FAAS.

\section{Agradecimentos}

Os autores agradecem à FAPESP pelo suporte financeiro e ao CNPq pela bolsa concedida.

Recebido em: 29/03/05

Aceito em: 23/05/05

F. A. Lobo, A. C.Villafranca, A. P. de Oliveira, M. de Moraes.TS-FF-AAS system with air-acetylene flame as alternative in relation to nitrous oxide-acetylene by FAAS for tin determination.

\begin{abstract}
This work propose the development of analytical procedure for on line tin determination in analytical solutions by thermospray flame furnace atomic absorption spectrometry (TS-FF-AAS) as alternative to nitrous oxide-acetylene flame by atomic absorption spectrometry, whose oxidant presents high costs with respect to air. Parameters were evaluated for the optimization of the system: flow of the carrier (air), volume of injected sample and concentration of the acid $(\mathrm{HCl})$. The linear analytical curve $[\mathrm{A}=$ $-0,00163+0,00319 \mathrm{Sn}],(\mathrm{r}=0,9998)]$ was constructed on interval of 10 the $80 \mathrm{mg} \mathrm{L}^{-1} \mathrm{Sn}$. The detection limit, the relative standard deviations $(\mathrm{n}=12)$ and analytical frequency were: $1,7 \mathrm{mg} \mathrm{L}^{-1} \mathrm{Sn}, £ 2,7 \%$ and 120 $\mathrm{h}^{-1}$, respectively. The TS-FF-AAS is an alternative for the determination of Sn.
\end{abstract}

Keywords: TS-FF-AAS; tin; metallic tube.

\title{
Referências
}

[1] E.C. Ferreira, K.G. Fernandes, A. R. A. Nogueira, J. A. Nóbrega, Resumos: 8th Rio Symposium on Atomic Spectrometry, MON 26, Paraty, RJ, 2004.

[2] A. P. Oliveira, Determinação direta e simultânea de Al, As, $\mathrm{Cu}, \mathrm{Fe}, \mathrm{Mn}, \mathrm{Ni}$, em álcool etílico hidratado combustível por Espectrometria de absorção atômica em forno de grafite. 2002. 93f. Dissertação (Mestrado em Química) - Instituto de Química, Universidade Estadual Paulista, São Paulo, 2002.
[3] J. Dëdina, D. L. A. Tsalev, Hydride Generation Atomic Absorption Spectrometry, John Wiley \& Sons, New York, p. 526, 1995.

[4] A.C. Villafranca, M. Moraes, J. Neira, Resumos: XVII Congresso Latino Americano de Química e 27 Reunião Anual da Sociedade Brasileira de Química, QA 261, Salvador, BA, 2004. [5] A. Gáspár, H. Berndt, Spectrochim Acta - Part B. 55 (2000) 587. 
[6] E. R. Pereira-Filho, H. Berndt, M. A. Z. Arruda, J. Anal. At. Spectrom. 17 (2002) 1308.

[7] A.Gáspár, É.SzéleS, H. Berndt, Anal. Bioanal. Chem. 372 (2002) 136.

[8] J. Davies, H.Berndt, Anal. Chim. Acta 479 (2003) 215.

[9] C. Nascentes, M. A. Z. Arruda, A. R. A. Nogueira, J. A. Nóbrega, Talanta 64(4) (2004) 912.

[10] M. G. Pereira, E. R. Pereira-Filho, H. Berndt, M. A. Z. Arruda, Spectroc. Acta Part B 59 (2004) 515.

[11] E. Gonzáles, R. Ahumada, V. Medina, Química Nova 27(6) (2004) 873.

[12] C.R.T. Tarley, M.A.Z. Arruda, Analytical Sciences 20 (6) (2004) 96.

[13] A. C. Villafranca, Avaliação da espectrometria de absorção atômica com nebulização térmica em tubo aquecido em chama (TS-FF-AAS) para determinação de $\mathrm{Cd}, \mathrm{Cu}, \mathrm{Pb}$ e $\mathrm{Zn}$ em álcool combustível e óleo diesel. 2004. 144f. Tese (Doutorado em Química) - Instituto de Química, Universidade Estadual Paulista, Araraquara, 2004.

[14] L. S. Guinese, Complexos Metálicos com 8Hidroxiquinolinato: Estudo do comportamento térmico e aspectos analíticos na utilização como precursores na produção De oxidos Mmstos do tipo TiSno x. 1998. 211f. Dissertação (Mestrado em Química) - Instituto de Química, Universidade Estadual Paulista, Araraquara,1998.

[15] F. A. Lobo, Desenvolvimento de sistemas de injeção em fluxo para a determinação de estanho por espectrometria de absorção atômica com forno aquecido na chama, em amostras alimentícias enlatadas. 2005. 110f. Dissertação (Mestrado em Química) - Instituto de Química, Universidade Estadual Paulista, Araraquara, 2005. 